\title{
Nasolabial cyst: A Rare Case report
}

\author{
Dr C R Sobhana ${ }^{1}$, Dr Suresh Babu ${ }^{2}$, Dr Sanoj N M ${ }^{3}$ \\ ${ }^{1}$ (Professor \& HOD, Department Of Maxillofacial Surgery, Government Dental college Trivandrum,India) \\ ${ }^{2}$ (Additional professor, Department Of Maxillofacial Surgery, Government Dental college Trivandrum,India) \\ ${ }_{3}^{3}$ (Post Graduate, Department Of Maxillofacial Surgery, Government Dental college Trivandrum,India)
}

\begin{abstract}
The nasolabial cyst is a rare non-odontogenic cyst originating in maxillofacial soft tissues These cysts are uncommon lesions located near the alar cartilage and extend to the inferior nasal meatus, superior alveo labial groove and floor of the nasal vestibule. These cyst appear as a swelling in the canine fossa, upper lip, gingivolabial sulcus, nasal alae and nasal vestibule ${ }^{7}$. With increasing size, nasoalveolar cyst may impinge on the antero inferior turbinate and push against the septum. Treatment consists of enucleation being preformed through intraoral access, with an incision in the vestibular sulcus. As the cyst walls are intimately related to the mucosa of the nasal floor in the majority of cases, the surgical maneuver may result in tearing of this mисоsa. In such cases suturing must be performed with absorbable suture in order to prevent the development of an oronasal communication. Recurrence is rare and the prognosis excellent.
\end{abstract}

Key words: Nasolabial cyst, maxillary cysts, non-odontogenic cyst, developmental cyst, soft tissue cyst, Klestadt cyst

\section{Introduction}

The Nasolabial cyst is a rare non-odontogenic cyst originating in maxillofacial soft tissues. ${ }^{5}$ These cysts are uncommon lesions located near the alar cartilage and extend to the inferior nasal meatus, superior alveolabial groove and floor of the nasal vestibule. ${ }^{14}$ The lesions cause painless swelling in sublabial fold, upper lips and sometimes cause nasal obstruction. Pain occurs when the cyst becomes infected. According to Allard ${ }^{14}$, this lesion was first described in 1882 by Zuckerkandl and since then two main etiological theories had been proposed. One holds that the lesion arises from entrapped nasolacrimal duct tissue ${ }^{1}$ and the other says that it is an embryonic fissural cyst. ${ }^{3}$ Klestadt $^{6}$ first postulated an embryologic origin for these cysts and considered that these lesions must originate from embryonic epithelium, entrapped in the developmental fissures between the lateral nasal and maxillary processes. These cyst appear as a swelling in the canine fossa, upper lip, gingivo labial sulcus, nasal alae and nasal vestibule ${ }^{9}$. With increasing size, Nasoalveolar cyst may impinge on the antero inferior turbinate and push against the septum. Longstanding pressure may cause erosion of the nasal floor or pre-maxilla. ${ }^{15}$ Patient's primary complaints are deformity and nasal obstruction. Clinically, these nonodontogenic cysts present as smooth, fluctuant soft-tissue masses between the upper lip and nasal aperture with obliteration of the nasolabial fold and elevation of the nasal ala. Bilateral cysts are rare. Slow painless enlargement of the swelling may develope over several years, but patients may present with an acutely painful swelling if the cyst becomes infected. Less commonly, extension of infection from these cysts may mimic facial cellulitis, periodontal abscess, acute maxillary sinusitis or a nasal furuncle. Treatment consists of enucleation preformed through intraoral access, with an incision in the vestibular sulcus. As the cyst walls are intimately related to the mucosa of the nasal floor in the majority of cases, the surgical maneuver may result in tearing of this mucosa. In such cases suturing must be performed with absorbable suture in order to prevent the development of an oronasal communication. Recurrence is rare and the prognosis excellent. Nevertheless, one must be careful in order to avoid perforation and collapse of the lesion. ${ }^{8}$ The purpose of this paper was to review the literature and discuss the histopathology and etiology of this condition and treatment of this pathology by surgical excision

\section{Case Report}

A 26 year old female patient reported to Deparment of Oral and Maxillofacial Surgery OPD with complaints of swelling near left side of ala of nose 2 weeks duration. During her routinue dental visits the surgeon noticed this swelling and referred to Govt Dental College, Trivandrum. Extra oral examination revealed a small swelling of $(1 \times 1 \mathrm{~cm})$ near the left ala of nose. Lesion was painless, without obliterating nasolabial fold and slight elevating the left ala of nose, soft in consistency, mobile and compressible extra orally, no palpable pulsation and rise in temperature in overlying skin. 


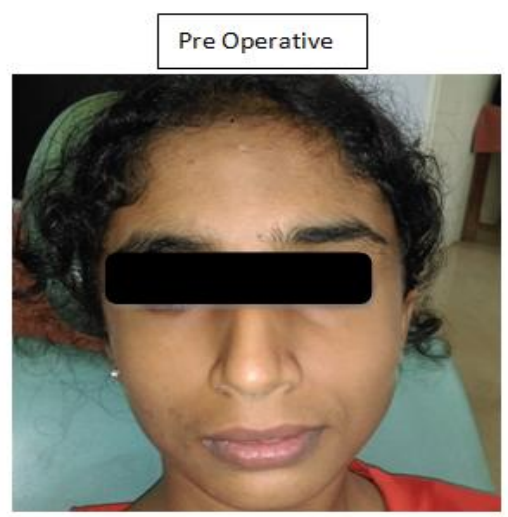

On Intra oral examination a $(1 \times 1 \mathrm{~cm})$ dome shape swelling was present in left labial vestibule in relation to 21,22. Lesion did not cross the midline. Labial frenum, gingiva and overlying mucosa appears to be normal in colour and consistency, no pus discharge, secondary changes like suppuration or ulceration and no caries and peri apical lesion. Swelling was soft, fluctuant, mobile in nature and related teeth were vital. Aspiration yielded only yellow clear fluid without cholesterol crystals.

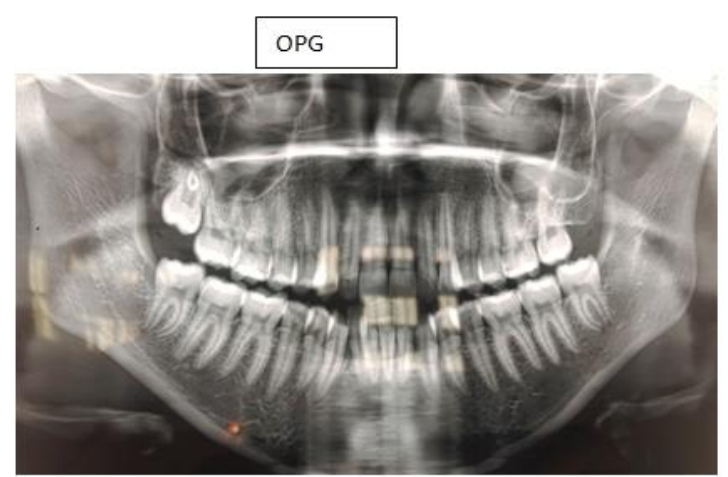

Radiographic examination included OPG, Occlusal, IOPAR failed to show bone involvement and tooth related pathology .USG revealed well defined cystic lesion with thick internal echoes and underlying bone appeared normal. Based on history, clinical examination and radiological findings, provisional diagnosis of nasolabial cyst was made. Excisional was performed through vestibular approach under LA .The lesion was soft, globular and removed in toto without nasal mucosal tear.

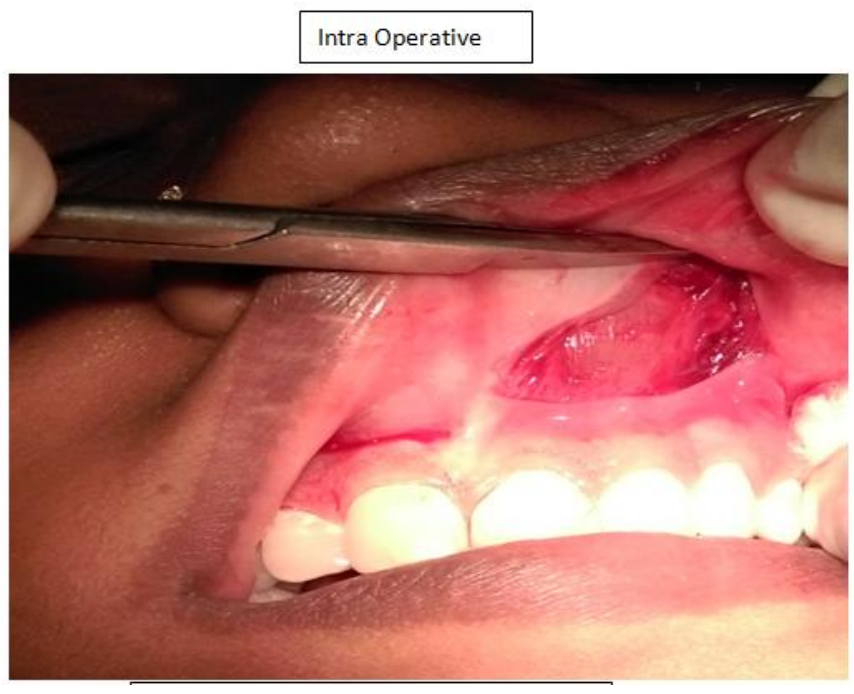




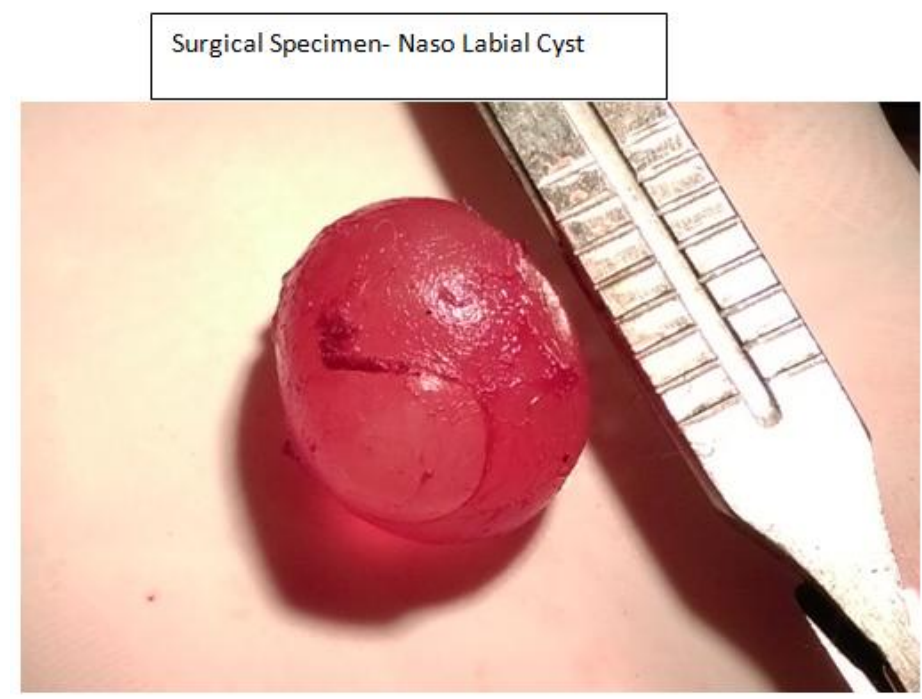

Histopathological examination revealed pseudo stratified ciliated columnar epithelium with numerous globlet cells. Post operative period was uneventful. All adjacent teeth remained vital and patient is under regular follow up.

\section{Discussion}

The nasolabial cyst is a rare non-odontogenic cyst originating in the maxillofacial soft tissues ${ }^{5}$. These are uncommon lesions located near the alar cartilage and extending into the inferior nasal meatus, superior alveolabial groove and floor of the nasal vestibule. ${ }^{14}$ These lesions are painless swellings in sublabial fold, upper lips and cause nasal obstruction. Pain occurs when the cyst becomes infected. Nasolabial cysts were first described by Zuckerkandl. ${ }^{14}$ The term Nasolabial cyst itself was coined by Rao, ${ }^{1}$ which was also been reported as Nasoalveolar cyst, Nasal vestibular cyst, Mucoid cyst of the nose, and Klestadt's cyst. The incidence of the cyst is $0.7 \%$ in overall cysts. ${ }^{3}$ Nasolabial cyst is mostly unilateral and is more commonly located on left side and more frequently in women. ${ }^{5,13,18}$ Bilaterality is reported in about $10 \%$ of patients ${ }^{7,9}$ and higher incidence in individuals of the black race. ${ }^{12,16}$ In the sample of van Bruggen et al. (1985) 119 patients were women (79\%) and 32 were men $(21 \%)$ a ratio of 3.7: 1. The nasolabial cyst occurs outside the bone in the nasolabial folds below the alae nasi. It is traditionally regarded as a jaw cyst although strictly speaking it should be classified as a soft tissue cyst. As the alveolus is not involved, the term nasolabial is preferred to naso alveolar cyst.

There are three theories for the formation of the cyst.

(1) The cyst is formed embryologically by retention epithelial cells in the maxilla \& medial and lateral nasal walls.

(2) The cyst is formed embryologically by retention cells from the inferior nasolacrimal channel cells.

(3) Exposure to trauma accelerates the formation of the cyst ${ }^{17}$.

The most frequent symptom is swelling and very often this was the only complaint. Sometimes the patients complain of pain and difficulty in nasal breathing but pain is generally present when the cysts are infected. In some cases, difficulty with an upper denture has drawn attention to the problem, and occasionally the cysts are diagnosed during routine examination. Cohen and Hertzanu (1985) reported a case that reached a huge size, causing severe facial deformity. In most cases the cysts are unilateral, but in the study of van Bruggen et al. ${ }^{12}$ patients had bilateral lesions $(10.6 \%)$. El-Hamd ${ }^{14}$ reported that complications when they occur, generally cause nasal block. Cohen \& Hertzanu ${ }^{13}$ have stated that patients only seek therapy when there is deformity, nasal block or infection caused by this lesion. The cysts grow slowly, producing a swelling of the lip. They fill out the nasolabial fold and often lift the ala nasi, distort the nostril and produce a swelling of the floor of the nose. Intra-orally they form a bulge in the labial sulcus. The cysts are fluctuant and on bimanual palpation, fluctuation may be elicited between the swelling on the floor of the nose and that in the labial sulcus. Infected cysts may discharge into the nose. The traditional concept of the pathogenesis of the nasolabial cyst was that is the soft tissue equivalent of the globulomaxillary cyst. It was therefore suggested that it arise from epithelium at the site of fusion of the globular, lateral nasal and maxillary processes. The differential diagnosis of nasolabial cysts include odontogenic cysts, periapical abscesses and periapical granulomas. Vitality testing of the adjacent teeth is essential for proper diagnosis as a coincidental lesion being absent and they will be vital in cases of nasolabial cyst. Dermoid and epidermoid cysts should also be considered in the differential diagnosis although they are associated with yellowish discoloration of the overlying mucosa, whereas in nasolabial cysts, the 
mucosa conserves its normal pink hue or appears blue-tinged. ${ }^{5}$ Dermoid and epidermoid cysts are usually diagnosed in childhood while nasolabial cysts are more common in adult patients. ${ }^{17}$ Seward (1962), RoedPetersen (1969), Kitamura (1976), Allard (1982) and David and O'Connell (1986) have reviewed the various hypotheses proposed to explain the origin of nasolabial cysts, and there is considerable support for the proposal first put forward by Brüggemann (1920) that they developed from the lower anterior part of the nasolacrimal duct. When the margins of the lateral nasal and maxillary bulges fused to form pre maxilla, the ectoderm along the boundary between them gives rise to a solid cellular rod which at first develops as a linear surface elevation, the nasolacrimal ridge, and then sinks into the mesenchyme and detaches from the overlying ectoderm. Its caudal end proliferates to connect with the caudal part of the lateral nasal wall while its cranial extremity later connects with the developing conjunctival sac. This solid rod then becomes canalised to form the nasolacrimal duct (Warwick and Williams, 1973; Sadler, 1995). The location of nasolabial cysts is such that they could conceivably develop from remnants of the embryonic nasolacrimal duct, if not from the lower anterior portion of the mature duct. The mature nasolacrimal duct is lined by pseudostratified columnar epithelium and this is the type of epithelium usually found lining nasolabial cysts.

A detailed description of the radiological features has been provided by Seward (1962). He pointed out that there was a localised increase in radiolucency of the alveolar process above the apices of the incisor teeth. This radiolucency resulted from a depression on the labial surface of the maxilla which may be detectable in a tangential view. When the depression extended to the lateral margin of the anterior bony aperture of the nose, there was resorption of the lower part of the nasal notch. The inferior margin of the anterior bony aperture of the nose was distorted by the lesion. As a result, standard occlusal radio-graphs showed a pronounced posterior convexity in half of the bracket shaped radiopaque line which formed the bony border of the nasal aperture, instead of the usual double curve. Chinellato and Damante (1984) confirmed from their own studies that this landmark feature was stable and could be identified in $90 \%$ of the cases except in the event of maxillary erosion, nasolabial cysts are undetectable on plain radiography, as they only contain soft tissue. Although the nasolabial cysts are extra-osseous they lie sub periosteally and careful surgical enucleation via sublabial approach is the treatment of choice. Su et al. (1999) reported on a transnasal endoscopic marsupialisation technique that they developed to treat 16 patients with nasolabial cysts.

Most studies have reported enucleation as the treatment of choice for nasolabial cysts. ${ }^{5,12}$ As they are entirely composed of soft tissues, nasolabial cysts are unresponsive to marsupialization. ${ }^{17}$ Alternative treatment modalities have been suggested, including aspiration, injection of sclerosing agents, and incision and drainage; however, these methods are associated with high recurrence rates. ${ }^{4,18,19}$ Therefore, in view of the ease of surgical exicision and its curative potential enucleation is the method of choice.

\section{Conclusion}

Based on the review of literature and the characteristics of the present case, it is concluded that, despite the low frequency of nasolabial cysts, it is essential that dental practitioners should be able to recognize the features of these lesions so as to be able to distinguish them from lesions of odontogenic origin and thus enable safe and proper treatment planning. Nasolabial cyst must be kept in mind with the differential diagnosis of swellings present at nasal vestibule, nasal base, and sublabial area.Such key features may be difficult to detect because patients may be asymptomatic, but most exhibit well-circumscribed swelling, localized pain, and partial or complete nasal obstruction on the affected side. Routinue radiographs like IOPAR, Occlusal, OPG fail to show such lesions. So other radiological measures like USG,CT,MRI are needed to find out extension of lesion. Histopathology is necessary to confirm the diagnosis. The optimal treatment modality is complete excision of the lesion which results in good prognosis and rare recurrence.

\section{Reference}

[1]. Zuckerkandl E. Normale und pathologische Anatomie der Nasenhohle. Vienna: W. Braunmuller; 1882

[2]. Klestadt WD. Nasal cyst and facial cleft cyst theory. Ann Otol Rhinl Laryngol 1953;62:84-89.

[3]. Rao RV. Nasolabial cyst. J Laryngol Otol. 1955;69:353-4.

[4]. Crawford Jr WH, Korchin L, Greskovich Jr FJ. Nasolabial cysts:report of two cases. J Oral Surg. 1968;26:582-8.

[5]. Allard rhb. Nasolabial cyst. A review of the literature and report of cases. Int J Oral Surg. 1982; 11:351-359.

[6]. Wesley RK, Scannel T, Nathan LE. Nasolabial cyst: Presentation of a case with a review of the literature. J Oral Maxillofacial Surg 1984; 42:188-192

[7]. Barzilai M. Case report: bilateral nasoalveolar cysts. Clin Radiol 49:140-141, 1994.

[8]. Cantisano, MH, Soubhia AMP, Tucci R, Zambon RLD. Cisto nasolabial: revisão de literatura e relato de um caso clínico. Rev Ciênc Odontol. 1998; 1(1): 27-30.

[9]. K. El-Din and A. A. el-Hamd, "Nasolabial cyst: a report of eight cases and a review of the literature," Journal of Laryngology and Otology, vol. 113, no. 8, pp. 747-749, 1999.

[10]. El-Hamd KEAA. Nasolabial Cyst: a report of eight cases and a review of the literature. J Laryngol Otol 1999;113:747-9.

[11]. El-Hamd KEAA. Nasolabial cyst: a report of eight cases and a review of the literature. J Laryngol Otol. 1999; 113: 747-9.

[12]. el-Din K, el-Hamd AA. Nasolabial cyst: a report of eight cases and a review of the literature. J Laryngol Otol. 1999;113: 747-9.

[13]. Pruna X. et al. Value of sonography in the assessment of space-Occupying of the anterior nasal fossa. J Clin Ultras 2000;28:14-9. 
[14]. Nixdorf DR, Peters E, Lung KE. Clinical presentation and differential diagnosis of nasolabial cyst. J Can Dent Assoc. 2003;69:1469.

[15]. Liu ES, Kridel RW. Evaluation of nasoalveolar cysts for the plastic surgeon. Arch Facial Plast Surg 5:185-188, 2003.

[16]. Nixdorf DR, Peters E, Lung KE.Clinical presentation and differential diagnosis of nasolabial cyst. J Can Dent Assoc. 2003; 69(3): 146-9

[17]. Erkan NA, Yilmazer C, Yilmaz I, Bolat FA. Nasoalveolar cysts: review of 3 cases. J ORL. 2005;67:196-8.

[18]. Ben Slama L, Zaghbani A, Hidaya S. Nasolabial cyst. Rev Stomatol Chir Maxillofac. 2009; 110: 338-9. http://dx.doi.org/10.1016/j. stomax.2009.09.004

[19]. Bettoni CH, Jaeger F, López-Alvarenga R, Rezende FO. Cisto nasolabial: revisão da literatura e relato de caso clínico. Rev Port Estomatol Med Dent Cir Maxilofac. 2011;52:157 